\title{
Novel approach for calibration breakdown voltage of large area SiPM
}

\author{
Sergei Dolinsky ${ }^{1}$ \\ GE Global Research Center \\ One Research Circle, Niskayuna NY, USA \\ E-mail: dolinsky@ge.com
}

Silicon photomultipliers (SiPMs) have a very good single photoelectron pulse (SPE) response that can be used for absolute calibration and defining the breakdown voltage. Unfortunately with an increasing size of SiPM it becomes more difficult to measure the SPE pulse due to pile up from the increased dark counts. Also this SPE calibration method requires a high gain low noise amplifier, which makes it impossible to use readout electronics designed for detection the relatively large signals from scintillators.

In this work we propose a novel method for calibrating SiPMs using a high intensity light pulse. The duration of pulse must be much smaller than the recovery time of the SiPM and the intensity of light pulse must be chosen large enough to trigger all micro-cells simultaneously ( 10-30 primary e-h/micro-cell/pulse). In this case, the signal is proportional to the gain and number of cells in the device and a nearly linear function of overvoltage even close to the breakdown voltage. Since the amplitude of the pulse is large, a preamplifier is not needed even when the SiPM operates as a regular avalanche photodiode below the breakdown voltage. Also this method allows investigation of an SiPM operating in the linear and avalanche (non-Geiger regime) modes and can be very useful for the study of the breakdown avalanche process and transitions to the Geiger mode.

International Workshop on New Photon-detectors,

LAL Orsay, France

June 13-15, 201

\footnotetext{
1 Speaker
} 


\section{Introduction}

SiPMs have become a very popular photosensor for scintillator based nuclear detectors. As is well known, the gain of the SiPM operating in Geiger mode is proportional to the overvoltage $\left(\mathrm{V}_{\mathrm{ov}}\right)$, the difference between the applied bias $\left(\mathrm{V}_{\text {bias }}\right)$ and breakdown voltages $\left(\mathrm{V}_{\mathrm{br}}\right)$. The Geiger mode process results in a well defined single photoelectron (SPE) pulse. These SPE pulses are very often used for calibration purposes to identify $\mathrm{V}_{\mathrm{br}}$ and the capacitance of individual microcells $\left(\mathrm{C}_{\text {micro-cell }}\right)[1]$. Two different approaches are utilized to detect SPE pulses: intrinsic dark counts or very short light pulses with an average of 0.1 to 10 detected photons. This method works well for small devices $\left(\sim 1 \mathrm{~mm}^{2}\right)$ and when the dark count rate is small. To build a nuclear detector for many applications, e.g. a PET scanner, one would like to use bigger SiPMs with a typical size of $10-100 \mathrm{~mm}^{2}$ [2]. Due to increased dark counts it becomes very difficult to use SPE pulses for calibration of these SiPMs. For example Fig.1 depicts oscilloscope snapshots for an HPK device $3 \times 3 \mathrm{~mm}^{2}$ and $6 \times 6 \mathrm{~mm}^{2}$ (MPPC-S10985-050C).

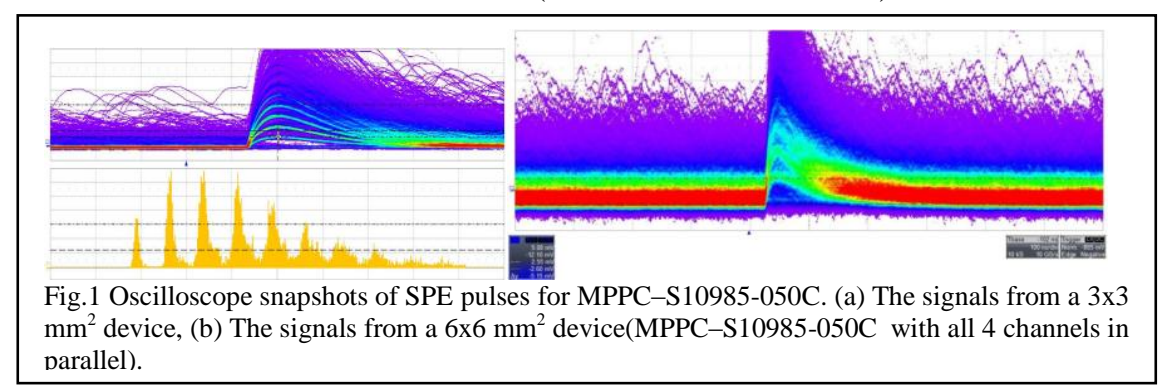

At small $\mathrm{V}_{\mathrm{ov}}$ the gain of the SiPM is small and the contribution of the preamplifier noise is significant. This makes it difficult to measure the pulses corresponding to $1 \mathrm{SPE}$ at small $\mathrm{V}_{\mathrm{ov}}$. At higher $\mathrm{V}_{\mathrm{ov}}$ the increased dark counts and the high probability of afterpulses make it nearly impossible to isolate SPE events. It is worth to notice that the SPE calibration method is very important for PDE measurements because it is the only method not sensitive to optical crosstalk between micro-cells and frequently used for device evaluation. The typical signals of scintillator based nuclear detectors correspond to 100-10000 photons and the readout electronics is optimized for this dynamic range[2]. Therefore the SPE method can not be used to calibrate a fully assembled detector using the same electronics. Even if the ASIC has a special high gain mode for the calibration, the high dark count rate presents a challenge to use SPE pulses to calibrate large area SiPMs. In this work we suggest a new approach for the calibration and characterization of SiPMs. This method is based on using a short light pulse with a relatively high intensity to saturate and trigger all the micro-cells of the SiPM simultaneously. With this method a large area SiPM can be calibrated without any difficulties because the amplitude of the pulse is approximately given by:

$$
\mathrm{V}_{\text {ampl }}=\mathrm{V}_{\text {ov }} \cdot \mathrm{R}_{\text {load }} /\left(\mathrm{R}_{\text {load }}+\mathrm{R}_{\text {quench }} / \mathrm{N}_{\text {cell }}\right)
$$

which can be a few hundred $\mathrm{mV}$ at $50 \Omega$ termination. Thus the fully assembled detector can be calibrated since the amplitude of the calibration signals are comparable with the signal due to scintillator pulses. 


\section{Comparison of low and high light intensity calibration}

\subsection{Low light intensity method}

As previously mentioned the calibration method based on a weak light source is often used for the characterization and calibration of an SiPM. The intensity of light pulses is adjusted to trigger on average 0.1 to 1 micro-cell per pulse. It is not neccessary to use a calibrated light source for the gain and relative PDE measuremnts. The events corresponding to $0,1,2$ and more fired micro-cells can be identified and used for the gain calibration and measurement of the Geiger mode probability. Fig. 2 presents results of $\mathrm{V}_{\mathrm{br}}$ calibration and relative PDE measurement for MPPC-S10985-050C, S/N 407 from Hamamatsu used for this study.

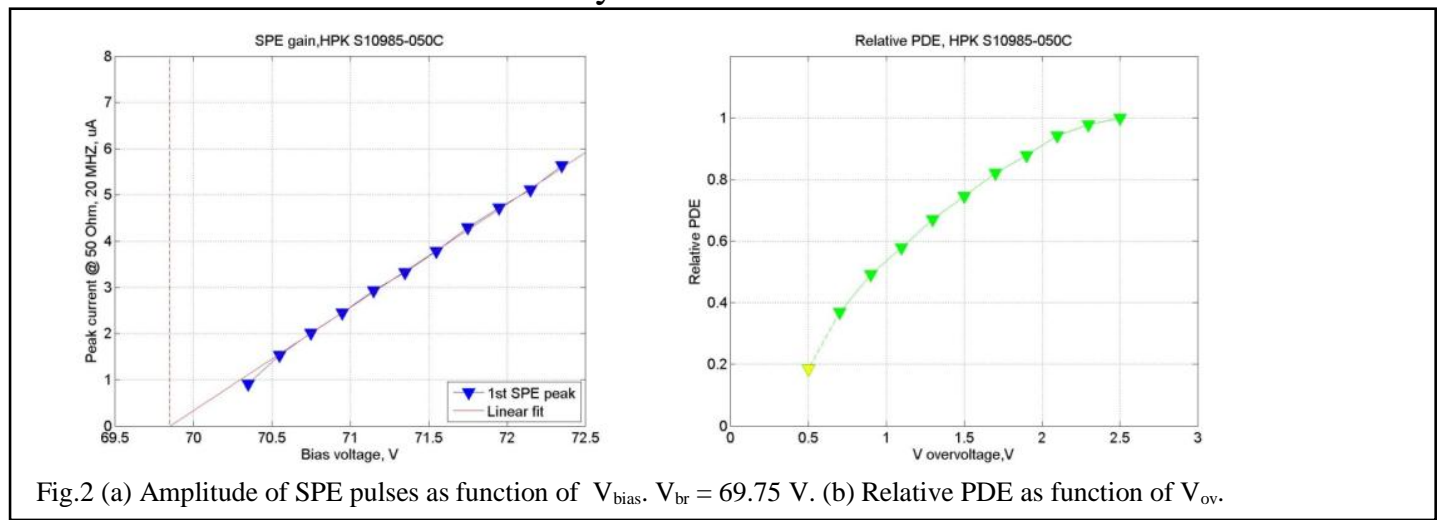

Due to the small signals and the contributions of electronic noise it is difficult to measure signals for $\mathrm{V}_{\text {ov }}$ below $0.5 \mathrm{~V}$. Using an increased intesity of the light pulses (up to 10-100 detected photons per pulse) the measurement of the average amplitude for calibration leads to the other well known problems.

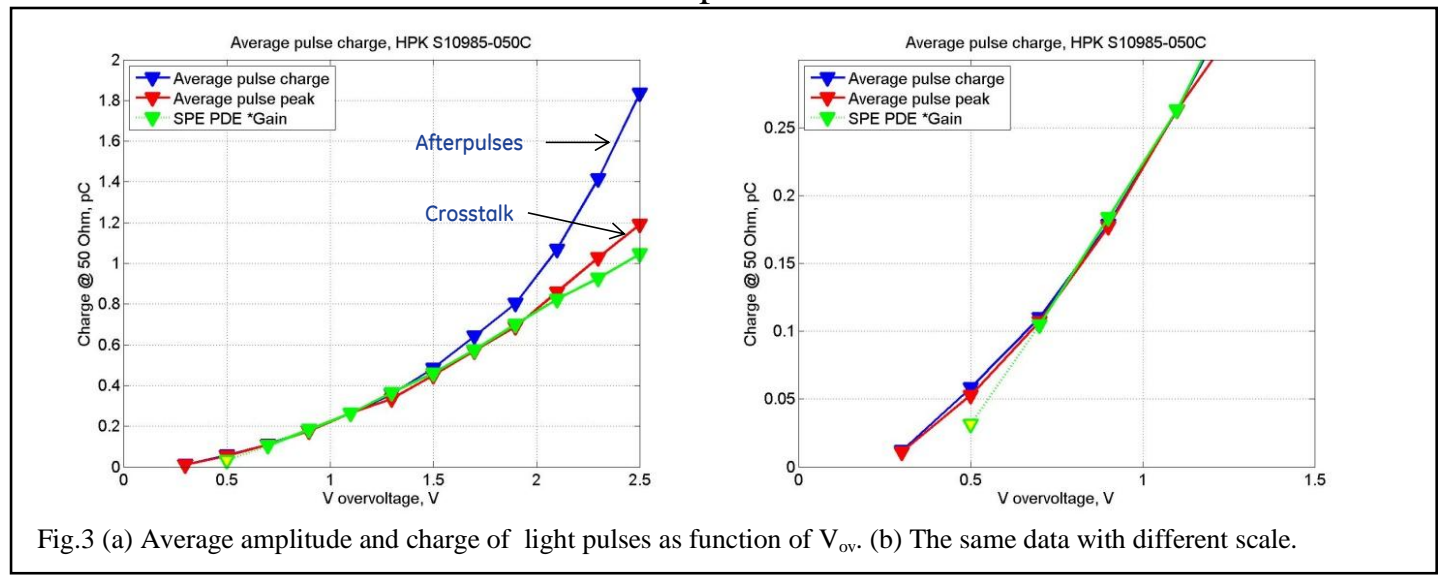

Figure 3 presents the results of the average pulse amplitude and charge measured for the same SiPM. The shape difference between the amplitude and charge graphs at $\mathrm{V}_{\mathrm{ov}}$ above $1.5 \mathrm{~V}$ is due to afterpulsing. Based on SPE gain and PDE measurements we can estimate the amplitude as function of $\mathrm{V}_{\mathrm{ov}}$. The deviation of measured amplitude from the predicted value at $\mathrm{V}_{\mathrm{ov}}>2 \mathrm{~V}$ can be used for an estimation of optical cross talk between the micro-cells. Since the PDE of SiPM depends on $\mathrm{V}_{\text {ov }}$ and diminishes around $\mathrm{V}_{\mathrm{br}}$, the average pulse height is relatively unsensitive around $\mathrm{V}_{\mathrm{br}}$ making it very difficult to reliably find $\mathrm{V}_{\mathrm{br}}$ from this measurement. 


\subsection{High light intensity method.}

We suggest to use a high intensity light source compare to the type of light sources used for calibration described previously. If during the short period of time the light pulse generates few electron-hole pairs per micro-cell the probability of triggering a Geiger mode avalanche can be represented as

$$
\mathrm{P}_{\text {Geiger }}\left(\mathrm{N}_{\mathrm{e}-\mathrm{h}}\right)=1-\left(1-\mathrm{P}_{\text {Geiger }}(1)\right)^{\mathrm{Ne}-\mathrm{h}}
$$

This probability increases significantly with the number of electron-hole pairs $\left(\mathrm{N}_{\mathrm{e}-\mathrm{h}}\right)$ and approaches $100 \%$ at even very small $\mathrm{V}_{\text {ov }}$. Since the avalanche process quenches when the voltage across the diode drops below $\mathrm{V}_{\mathrm{br}}$, the total charge and signal from individual micro-cell does not depend on $\mathrm{N}_{\mathrm{e}-\mathrm{h}}$. Figure 4 presents model results for micro-cell triggering probability and the expected signal amplitude. For this model we use the meausured PDE $\left(\mathrm{V}_{\mathrm{ov}}\right)$. Because we could not measure PDE at $\mathrm{V}_{\mathrm{ov}}<0.5 \mathrm{~V}$ we use a linear approximation to obtain its value at $\mathrm{V}_{\mathrm{br}}$.
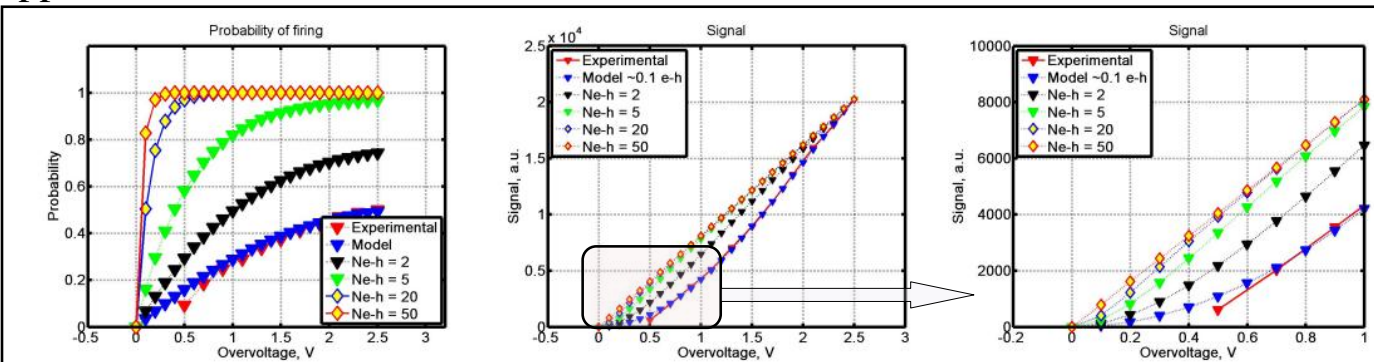

Fig.4 (a) Probability of triggering a Geiger mode avalanche as function of $\mathrm{V}_{\mathrm{ov}}$ for different $\mathrm{N}_{\mathrm{e}-\mathrm{h}}$. (b)Signal response of SiPM for different intensities of calibration light pulse. (c) The same data with a different scale to show the behavior around $\mathrm{V}_{\mathrm{br}}$.

Based on this simplified model the light pulses with $\sim 10-30$ initial $\mathrm{N}_{\mathrm{e}-\mathrm{h}}$ per micro-cell shoud provide linear behaviour for $\mathrm{V}_{\mathrm{ov}}>0.1 \mathrm{~V}$.

We used a fast pulsed laser (PicoQuant model LDH-D-C) for these measurements. The wavelength is $405 \mathrm{~nm}$ and maximum pulse energy is $50 \mathrm{pJ}$ per pulse. The laser beam was defocused to uniformly illuminate the SiPM sensitive area. The pulse energy is sufficient to produce $\mathrm{N}_{\mathrm{e}-\mathrm{h}}>50$ per micro-cell (50 um micro-cell) when uniformly illuminated The pulse repetition rate $10 \mathrm{kHz}$ was used so the SiPM will be rechared between pulses and the average current through the device is sufficiently low to mimimize any temperature change of the Si avalanche area. A Source Measure Unit (Keithley model 2400) is used to bias the SiPM and measure the current.

Figure 5 presents I-V measurements of SiPM for different laser intensities. Applying small $\mathrm{V}_{\text {bias }}(\sim 10 \mathrm{~V})$ we use SiPM in photodiode mode and measure the photocurrent with Gain $=1$. The number of intial e-h generated by light pulse can be calculated from the photocurrent from the formula:

$$
\mathrm{I}_{\mathrm{SiPM}}=\mathrm{N}_{\mathrm{e}-\mathrm{h}} \cdot \mathrm{Q}_{\mathrm{e}} \cdot \mathrm{N}_{\text {cell }} \cdot \mathrm{F}_{\text {rep }}
$$

Where $\mathrm{N}_{\mathrm{e}-\mathrm{h}^{-}}$is the number of initial e-h per pulse per micro-cell, $\mathrm{Q}_{\mathrm{e}}$ is the electron charge, $\mathrm{N}_{\text {cell }}$ is the number of micro-cells per SiPM and $\mathrm{F}_{\text {rep }}$ is the frequency of the calibration pulses. We use Hamamatsu $3 \times 3 \mathrm{~mm}^{2}$ device with $\mathrm{N}_{\text {cell }}=3600$, so the photocurrent corresponding to $\mathrm{N}_{\mathrm{e}-\mathrm{h}}=30$ per micro-cell is $\mathrm{I}_{\mathrm{SiPM}}=0.17 \mathrm{nA}$.

It is interesting to note that there is no indication of afterpulsing at even high $\mathrm{V}_{\mathrm{ov}}$. This can be explained by the high value of $R_{\text {load }}(1 \mathrm{k} \Omega$ ) we use during these measurements. Because all the micro-cells of the SiPM are triggered simultaneously, the recharging current for all cells is limited by $\mathrm{R}_{\text {load. }}$. It can be shown that recharging time $\tau$ is: 


$$
\tau=\left(\mathrm{R}_{\text {load }} \cdot \mathrm{N}_{\text {cell }}+\mathrm{R}_{\text {quench }}\right) \cdot \mathrm{C}_{\text {micro-cell }}
$$

For this SiPM, the recharging time with $\mathrm{R}_{\text {load }}=1 \mathrm{k} \Omega$ is 325 ns. Because of this long recharging time the probability of afterpulses is low even at $\mathrm{V}_{\mathrm{ov}}=2 \mathrm{~V}$.

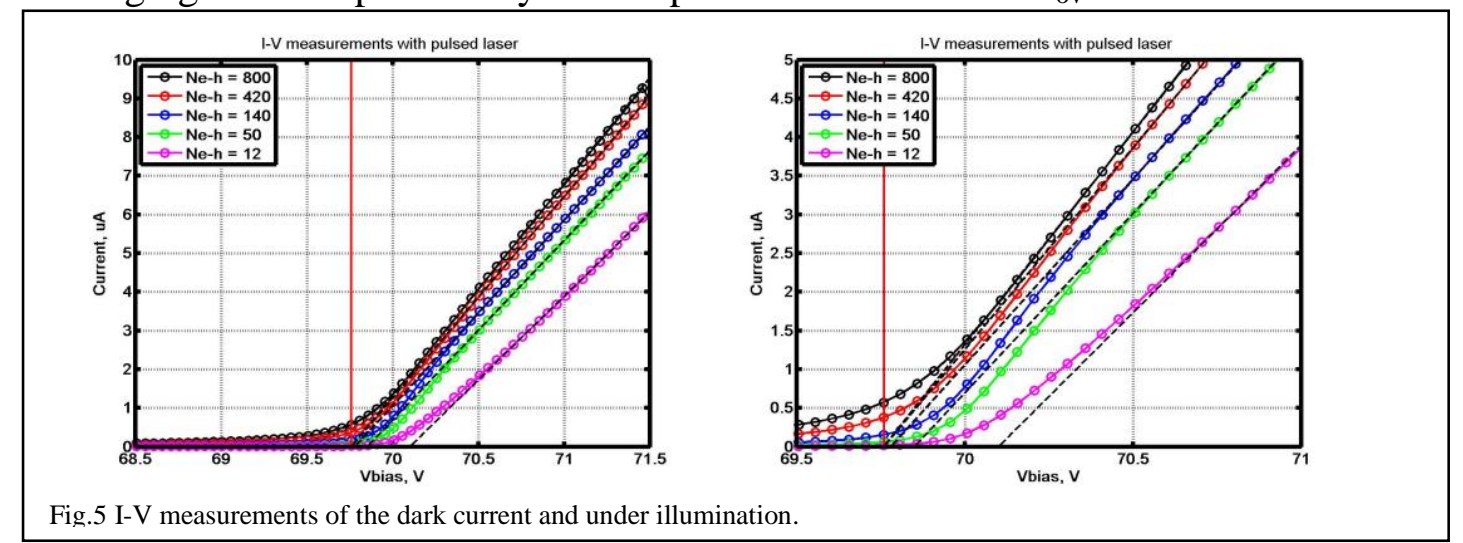

Below $\mathrm{V}_{\mathrm{br}}$ the SiPM operates as an avalanche photodiode and, as expected, above $\mathrm{V}_{\mathrm{br}}$ the current is a linear function of $\mathrm{V}_{\mathrm{ov}}$. In this simplified model the slope of the I-V curve should not depend on the number of initial e-h pairs and is given by:

$$
\mathrm{dI} / \mathrm{dV}=\mathrm{N}_{\text {cell }} \cdot \mathrm{C}_{\text {micro-cell }} \cdot \mathrm{F}_{\text {rep }}
$$

By measuring the slope $\mathrm{dI} / \mathrm{dV}$ we can calculate $\mathrm{C}_{\text {micro-cell. }}$ The results of $\mathrm{V}_{\mathrm{br}}$ and estimated $\mathrm{C}_{\text {micro-cell }}$ from a linear approximation of $\mathrm{I}-\mathrm{V}$ curves are presented in Table.1. The $\mathrm{V}_{\mathrm{br}}$ measurements are in good agreement with the calibration based on the SPE method for $\mathrm{N}_{\mathrm{e}-\mathrm{h}}>50$ and have a slightly higher values for smaller intensities. This deviation is similar to our modeling results for small $\mathrm{N}_{\mathrm{e}-\mathrm{h}}$ (fig.4(c)) and depend on the exact function of $\operatorname{PDE}\left(\mathrm{V}_{\mathrm{ov}}\right)$ for small $\mathrm{V}_{\mathrm{ov}}$.

The capacitance of the microcell provided by the manufacturer is 90 $\mathrm{fF}$. We found that $\mathrm{dI} / \mathrm{dV}$ value corresponds to $\mathrm{C}_{\text {micro-cell }}$ at small intensity $\left(\mathrm{N}_{\mathrm{e}-\mathrm{h}} \sim 10\right)$ but gives significantly higher values when the

\begin{tabular}{|c|c|c|c|}
\hline $\begin{array}{l}\mathrm{N}_{\text {e-h }} / \mathbf{u}- \\
\text { cell/pulse }\end{array}$ & $\begin{array}{l}\text { Effective } \\
\text { C }_{\text {u-cell }}(\mathbf{f F})\end{array}$ & $\mathbf{V}_{\mathrm{br}(\mathrm{V})}$ & dVpeak/dVbias \\
\hline 800 & $152 \mathrm{fF}$ & 69.76 & 0.9 \\
\hline 420 & $145 \mathrm{fF}$ & 69.76 & - \\
\hline 140 & $132 \mathrm{fF}$ & 69.78 & 0.75 \\
\hline 50 & $122 \mathrm{fF}$ & 69.85 & - \\
\hline 12 & $97 \mathrm{fF}$ & 70.05 & 0.6 \\
\hline
\end{tabular}
number of initial e-h increased.

\section{Pulse measurements}

As previously mentioned, when all the SiPM micro-cells are fired simultaneously the amplitude of the signal is large and the pulse measurements can be done directly with a $50 \Omega$ termination without amplification. Figure 6(a) represents typical signals from a Hamamatsu $3 \times 3 \mathrm{~mm}^{2}$ MPPC. For very high intensities, the pulses can be observed below $\mathrm{V}_{\mathrm{br}}$ when the SiPM operates in limited avalanche mode.

The amplitude and total charge for each pulse can be measured and Figure 6(b) presents pulse amplitude charge as function of $\mathrm{V}_{\text {bias }}$. At high $\mathrm{V}_{\text {bias }}$ the total charge deviates from linear behavior due to increased probability of afterpulses. Since for pulse measurements we use $\mathrm{R}_{\text {load }}=50 \Omega$ instead of $1 \mathrm{k} \Omega$ used before, the recharging time is shorter $\tau \sim 30 \mathrm{~ns}$ and the afterpulses start at lower $\mathrm{V}_{\mathrm{ov}}$. The afterpulse effect can be clearly seen above $\mathrm{V}_{\text {bias }}>71 \mathrm{~V}$ for $\mathrm{I}-\mathrm{V}$ curves. 


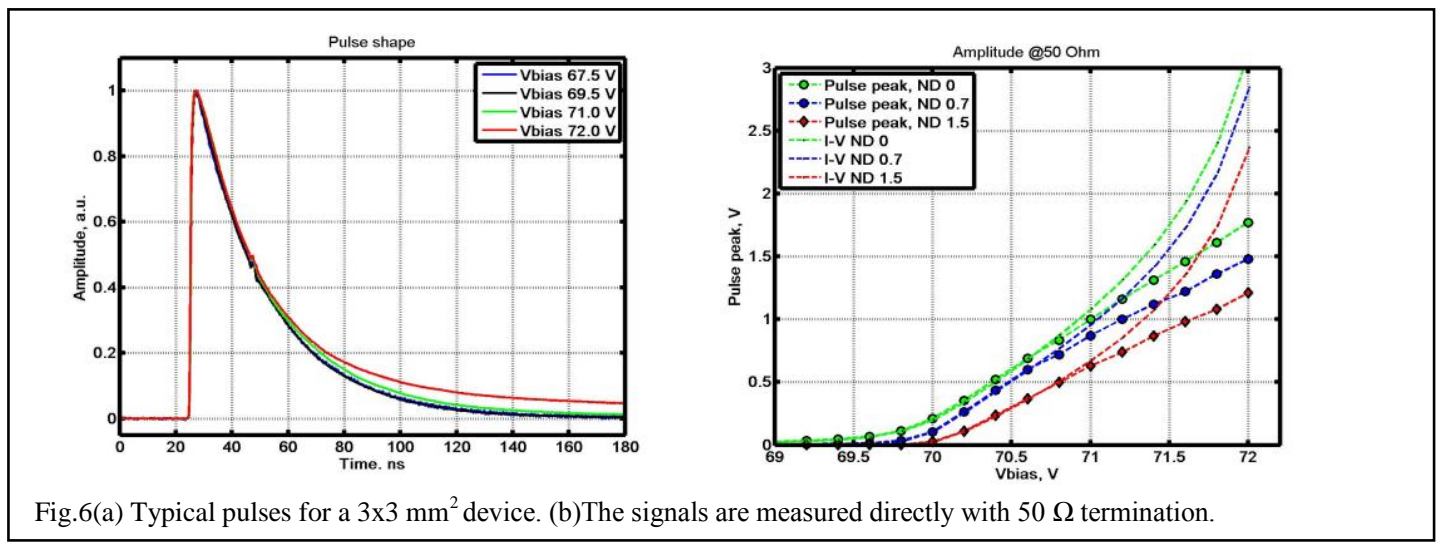

Without afterpulses the pulses at $\mathrm{R}_{\text {load }}$ can be expressed as

$$
\mathrm{V}_{\mathrm{Rload}}=\mathrm{V}_{\text {OV }} \cdot \mathrm{R}_{\text {load }} /\left(\mathrm{R}_{\text {load }}+\mathrm{R}_{\text {quench }} / \mathrm{N}_{\text {cell }}\right) \cdot \exp (-\mathrm{t} / \tau)
$$

With $\tau=\left(\mathrm{R}_{\text {load }} \cdot \mathrm{N}_{\text {cell }}+\mathrm{R}_{\text {quench }}\right) \cdot \mathrm{C}_{\text {micro-cell }}$. Any afterpulses do not change the peak amplitude but increase the charge at high $\mathrm{V}_{\mathrm{ov}}$. Figure 6(b) shows the pulse amplitude as function of $\mathrm{V}_{\mathrm{ov}}$ and I-V measurements for three different laser intensities. The pulse amplitude is linear function of $\mathrm{V}_{\mathrm{ov}}$ and is not affected by aftepulses. The slope of measured $\mathrm{V}_{\text {pulse }}\left(\mathrm{V}_{\text {bias }}\right)$ is given by:

$$
\mathrm{dV}_{\text {pulse }} / \mathrm{dV}_{\text {bias }}=\mathrm{R}_{\text {load }} /\left(\mathrm{R}_{\text {load }}+\mathrm{R}_{\text {quench }} / \mathrm{N}_{\text {cell }}\right)
$$

and should not depend on laser pulse intensity. The results of measured $d V_{\text {pulse }} / \mathrm{dV}_{\text {bias }}$ are presented in Table.1. The expected value for the slope is approximately 0.6 and in a good agreement with the measurement. At higher $\mathrm{N}_{\mathrm{e}-\mathrm{h}}$ the slope increases. The simple model of SiPMs operating in Geiger mode assumes that the micro-cell discharge and the total charge does not depend how many primary e-h initiate this avalanche. The microcell needs to be recharged through $\mathrm{R}_{\text {quench }}$ before it can be triggered again. This model works well up to $\mathrm{N}_{\mathrm{e}-\mathrm{h}}<\sim 10$ but can not explain the behavior of investigated SiPM for very high intensites.

\section{Conclusion}

In this work we demonstrated a novel calibration method for the breakdown voltage of large area SiPMs based on short high intensity light pulses. With the proper light pulse intensity this method is simple and robust and does not require high gain amplifiers. It can be used with front-end electronics designed for PET detectors. This method also allows the investigation of the linear and avalanche (non-Geiger regime) modes and can be very useful for study of the breakdown avalanche process and transition into the Geiger mode. We found that a simple model describing the avalanche process for the Geiger mode works only for moderate light pulse intensities.

\section{References}

[1] P. Buzhan, B. Dolgoshein et al. Advanced study of silicon photomultiplier, presented at ICFA, Volume 23, 2001. Available: http://www.slac.stanford.edu/pubs/icfa/

[2] C.Kim et al. Time-of-Flight PET-MR Detector Development with Silicon Photomultiplier, Nuclear Science Symposium (NSS/MIC), IEEE, M18-25, 2012 